\title{
The First Trimester Gravid Serum Regulates Procalcitonin Expression in Human Macrophages Skewing Their Phenotype In Vitro
}

\author{
Damiano Rami, ${ }^{1}$ Martina La Bianca, ${ }^{1}$ Chiara Agostinis, ${ }^{2}$ Giorgio Zauli, ${ }^{2}$ \\ Oriano Radillo, ${ }^{2}$ and Roberta Bulla ${ }^{1}$ \\ ${ }^{1}$ Department of Life Sciences, University of Trieste, Via Valerio 28, 34127 Trieste, Italy \\ ${ }^{2}$ Institute for Maternal and Child Health, IRCCS "Burlo Garofolo", 34137 Trieste, Italy \\ Correspondence should be addressed to Roberta Bulla; rbulla@units.it
}

Received 18 October 2013; Accepted 29 January 2014; Published 5 March 2014

Academic Editor: Anna Chelmonska-Soyta

Copyright (C) 2014 Damiano Rami et al. This is an open access article distributed under the Creative Commons Attribution License, which permits unrestricted use, distribution, and reproduction in any medium, provided the original work is properly cited.

Procalcitonin (PCT) is one of the best diagnostic and prognostic markers in clinical practice, widely used to evaluate the evolution of bacterial infections. Although it is mainly produced by thyroid, during sepsis almost all the peripheral tissues are involved in PCT production. Parenchymal cells have been suggested as the main source of PCT expression; however the contribution of macrophages is not clear yet. In response to environmental cues, tissue macrophages acquire distinct functional phenotypes, ranging from proinflammatory (M1) to anti-inflammatory (M2) phenotype. Macrophages at the fetal-maternal interface show immunosuppressive M2-like activities required for the maintenance of immunological homeostasis during pregnancy. This study aims to clarify the ability to synthesise PCT of fully differentiated (M0), polarized (M1/M2) macrophages and those cultured either in the presence of first trimester gravid serum (GS) or pregnancy hormones. We found out that M1 macrophages upregulate PCT expression following LPS stimulation compared to M0 and M2. The GS downregulates PCT expression in macrophages, skewing them towards an M2-like phenotype. This effect seems only partially mediated by the hormonal milieu. Our findings strengthen the key role of macrophages in counteracting inflammatory stimuli during pregnancy, suggesting PCT as a possible new marker of M1-like macrophages.

\section{Introduction}

Procalcitonin (PCT) is the prohormone of calcitonin (CT) and is composed of 116 amino acids with a molecular mass of about $14 \mathrm{kDa}[1]$. PCT is usually released into the circulation from neuroendocrine cells of the thyroid gland (parafollicular or C cells) [2] and the lungs (K cells) [3], reaching physiological concentrations lower than $0.05 \mu \mathrm{g} / \mathrm{L}$ in the plasma of healthy people [4]. During an inflammatory status, PCT derives from almost all cell types and tissues, including monocytes and parenchymal tissues, making its upregulation less dependent on one type of cell, tissue, or organ [5-8]. The synthesis of PCT is mainly stimulated through bacterial endotoxins lipopolysaccharides (LPS) [9] and, to lesser extent, from the proinflammatory cytokines IL$1 \beta$, IL-6, and TNF $\alpha[10,11]$. Its levels are however attenuated by the release of IFN $\gamma$ in response to viral infections [12]. PCT is now generally accepted as a good prognostic and diagnostic marker of bacterial infection, due to its high stability in serum and its ability to respond more rapidly to inflammatory stimuli than other laboratory parameters of infection, such as C-reactive protein (CRP) and TNF $\alpha$, making it particularly suitable for routine laboratory analysis [13]. Macrophages are an essential component of innate immunity and play a central role in inflammation and host defense $[14,15]$. In response to environmental cues, mononuclear phagocytes acquire distinct functional phenotypes, ranging from a "classical" proinflammatory/antiangiogenic (M1) to an "alternatively" anti-inflammatory/proangiogenic (M2) phenotype, which represents extremes of a continuum in a universe of different activation states [16, 17]. Diversity, plasticity, and flexibility are hallmarks of cells of the monocytes-macrophages lineage, 
either under physiological or pathological conditions. Therefore, the functional skewing of macrophages is one of the best mechanisms to modulate both the systemic and local immunity [18]. Macrophages play a key role in counteracting inflammation during pregnancy [19]. This state represents an intriguing immunological condition, in which an immunosurveillance and a repression of the immune system both coexist, thanks to which the mother is able to tolerate the presence of the foetus and to quickly respond to local and systemic inflammatory stimuli [20]. Up to now, there are several studies in the literature which show freshly isolated human monocytes expressing PCT $[8,11,21]$; on the other hand, the contribution of fully differentiated and polarized macrophages in PCT expression is not known yet. In this study we investigate the involvement of primary human macrophages in PCT production, evaluating the modulation of PCT expression in these immune cells during normal and inflammatory conditions; besides, we aim to show if the synthesis of this prohormone can be somehow regulated during the macrophage polarization towards M1 and M2 phenotypes. Moreover, based on the importance of counteracting inflammation during gestation, our study examines how the human serum of the first trimester of pregnancy (GS) modulates PCT expression in fully differentiated and polarized macrophages, analysing its capability to skew their phenotype in vitro. For the first time, we showed that PCT is expressed both at gene and protein level in fully differentiated macrophages, and it is upregulated in those with M1 phenotype under stimulation with the proinflammatory stimulus LPS. Moreover, the GS has the capability to downregulate PCT expression in human macrophages in vitro, skewing them into an M2-like phenotype.

\section{Materials and Methods}

2.1. Reagents and Antibodies. The human cytokines IL-4 and IFN $\gamma$ were purchased from ImmunoTools $\mathrm{GmbH}$ (Friesoythe, Germany) and IL-10 from PeproTech EC Ltd. (DBA Italia S.r.l, Milan, Italy). Ultrapure lipopolysaccharide (LPS) from E. coli 0111:B4 strain was obtained from InvivoGen (Labogen S.r.l., Milan, Italy). Polyclonal rabbit anti-human PCT was purchased from Abcam (ProdottiGianni, Milan, Italy). CD68 (Macrosialin, mouse anti-human monoclonal antibody, and clone EMB11) and CD206 (Macrophage mannose receptor 1, mouse anti-human monoclonal antibody, and clone MCA2155) antibodies were purchased, respectively, from Dako (Milan, Italy) and AbD Serotec (Milan, Italy). Secondary goat anti-mouse-Cy3 and streptavidin $\mathrm{Cy} 2-$ conjugated were purchased from Jackson ImmunoResearch (LiStarFish, Milan, Italy); biotinylated swine anti-rabbit IgG were from Dako. The first trimester gravid serum (GS) was obtained at gestational week 12 (normal pregnant women). A pool of fresh sera from healthy blood donors was used as normal human serum (NHS). The sera were heat-inactivated at $56^{\circ} \mathrm{C}$ for 30 minutes prior to use. An informed consent was obtained from all women participating in the study. The study was approved by the Bioethical Committee of IRCCS, Burlo
Garofolo, Trieste, Italy. All the samples were immediately centrifuged, aliquoted, and frozen at $-80^{\circ} \mathrm{C}$.

2.2. Isolation and Differentiation of Human Peripheral Monocytes into Macrophages and Their In Vitro Polarization. Peripheral blood mononuclear cells (PBMCs) from healthy blood donors were isolated from anonymous buffy coats, kindly provided by the local blood bank (Immunotransfusional Department, Maggiore Hospital, Trieste, Italy) using Ficoll-Paque Plus density gradient (GE Healthcare Euroclone, Milan, Italy). Residual T and B cells were removed from the monocyte fraction by plastic adherence, after incubation for 2 hours at $37^{\circ} \mathrm{C}$ and $5 \% \mathrm{CO}_{2}$ in RPMI-1640 GlutaMAX (Life Technologies, Milan, Italy) supplemented with $10 \%$ of NHS and 1\% Penicillin/Streptomycin (Sigma-Aldrich). Fully differentiated and M1- and M2-polarized macrophages were obtained by culturing $10^{6}$ monocytes $/ \mathrm{mL}$ for 7 days at $37^{\circ} \mathrm{C}$ and $5 \% \mathrm{CO}_{2}$ with the same medium above described and replaced twice a week. M1 cells were polarized by stimulating overnight $(\mathrm{O} / \mathrm{N})$ with LPS $(100 \mathrm{ng} / \mathrm{mL})$ (InvivoGen) and IFN $\gamma(500 \mathrm{U} / \mathrm{mL})$ (ImmunoTools), while M2 macrophages were polarized by stimulating $\mathrm{O} / \mathrm{N}$ with IL-4 $(20 \mathrm{ng} / \mathrm{mL})$ (ImmunoTools) and IL-10 (50 ng/mL) (PeproTech) [22, 23].

\subsection{Stimulation of Macrophages with First Trimester Gravid} Serum. To avoid the presence of cellular debris, the first trimester gravid serum (GS) was centrifuged at $10.000 \mathrm{~g}$ for 5 minutes at room temperature (RT) and then filtered with Costar Spin-X Centrifuge Tube Filters, $0.22 \mu \mathrm{m}$ Pore CA Membrane (Corning, Turin, Italy). Fully differentiated macrophages were incubated for 24 hours at $37^{\circ} \mathrm{C}$ and $5 \%$ $\mathrm{CO}_{2}$ in RPMI-1640 GlutaMAX (Life Technologies) supplemented with $10 \%$ of GS and 1\% Penicillin/Streptomycin (Sigma-Aldrich). Cells were then stimulated for 3 hours at $37^{\circ} \mathrm{C}$ and $5 \% \mathrm{CO}_{2}$ in the culture medium with $10 \%$ NHS and LPS at a final concentration of $100 \mathrm{ng} / \mathrm{mL}$, to induce the gene expression of PCT.

2.4. Stimulation of Macrophages with Progesterone, 17 $\beta$ Estradiol, and Human Chorionic Gonadotropin (hCG). Fully differentiated macrophages were incubated for 24 hours at $37^{\circ} \mathrm{C}$ and $5 \% \mathrm{CO}_{2}$ in RPMI-1640 GlutaMAX (Life Technologies) supplemented with $10 \%$ of NHS and $1 \%$ Penicillin/Streptomycin (Sigma-Aldrich) in the presence of $100 \mathrm{nM}$ of progesterone (PG), $10 \mathrm{nM}$ of $17 \beta$-Estradiol (E2), and $20 \mathrm{U} / \mathrm{mL}$ of human chorionic gonadotropin (hCG), hormones that have been used alone or combined all together. Cells were then stimulated for 3 hours at $37^{\circ} \mathrm{C}$ and $5 \% \mathrm{CO}_{2}$ in the culture medium with $10 \%$ NHS and $100 \mathrm{ng} / \mathrm{mL}$ of LPS. Concentrations of the pregnancy hormones were the same as that reached in the serum of women during the 12th week of pregnancy.

2.5. Viability Assay. Cell viability was evaluated by Trypan Blue (Sigma-Aldrich) dye exclusion test. No differences were observed before and after completing the experiments. 
TABle 1: Primer used for qPCR analysis.

\begin{tabular}{|c|c|c|c|c|c|}
\hline Gene & Primers & Sequence $5^{\prime} \rightarrow 3^{\prime}$ & Annealing temperature $\left({ }^{\circ} \mathrm{C}\right)$ & Amplicon size (bp) & Gene bank accession number \\
\hline \multirow{2}{*}{$18 \mathrm{~S}$} & For & ATCCCTGAAAAGTTCCAGCA & \multirow{2}{*}{60} & \multirow{2}{*}{154} & \multirow{2}{*}{ NM_022551 } \\
\hline & Rev & CCCTCTTGGTGAGGTCAATG & & & \\
\hline \multirow{2}{*}{ PCT } & For & TCTAAGCGGTGCGGTAATCTG & \multirow{2}{*}{60} & \multirow{2}{*}{85} & \multirow{2}{*}{ NM_001741 } \\
\hline & Rev & CAGTTTGGGGGAACGTGTGA & & & \\
\hline \multirow{2}{*}{ IL- $1 \beta$} & For & TTCCCTGCCCACAGACCTTC & \multirow{2}{*}{66} & \multirow{2}{*}{298} & \multirow{2}{*}{ NM_000576 } \\
\hline & Rev & AGGCCCAAGGCCACAGGTAT & & & \\
\hline \multirow{2}{*}{ IL-10 } & For & CCAAGCCTTGTCTGAGATGAT & \multirow{2}{*}{61} & \multirow{2}{*}{120} & \multirow{2}{*}{ NM_000572 } \\
\hline & Rev & CTGAGGGTCTTCAGGTTCTCC & & & \\
\hline \multirow{2}{*}{ CD80 } & For & AGGAACACССТCСАATCTCTG & \multirow{2}{*}{60} & \multirow{2}{*}{150} & \multirow{2}{*}{ NM_005191 } \\
\hline & Rev & GGTCAAAAGTGAAAGCCAACA & & & \\
\hline \multirow{2}{*}{ CD206 } & For & TATGGAATAAAGACCCGCTGAC & \multirow{2}{*}{61} & \multirow{2}{*}{133} & \multirow{2}{*}{ NM_002438 } \\
\hline & Rev & TGCTCATGTATCTCTGTGATGCT & & & \\
\hline
\end{tabular}

2.6. RNA Isolation, cDNA Synthesis, and Quantitative RealTime PCR ( $q P C R)$. RNA was purified from cells with EuroGOLD trifast (Euroclone) according to the manufacturer's instructions. Total RNA was extracted and reversetranscribed as previously described [24]. Quantitative RealTime PCR (qPCR) was carried out on a Rotor-Gene 6000 (Corbett, Qiagen, Ancona, Italy) using iQ SYBR Green Supermix (Bio-Rad, Milan, Italy). Table 1 shows the primer list used for qPCR. The melting curve was recorded between $55^{\circ} \mathrm{C}$ and $99^{\circ} \mathrm{C}$ with a hold every $2 \mathrm{~s}$. The relative amount of gene production in each sample was determined by the Comparative Quantification (CQ) method supplied as part of the Rotor Gene 1.7 software (Corbett Research) [25]. The relative amount of each gene was normalized with $18 \mathrm{~S}$ and expressed as arbitrary units (AU) considering 1 AU obtained from fully differentiated macrophage used as calibrator.

2.7. Immunofluorescence. Isolated PBMCs, obtained as previously described, were plated for 48 hours at $37^{\circ} \mathrm{C}$ and $5 \% \mathrm{CO}_{2}$ in RPMI-1640 GlutaMAX (Life Technologies) supplemented with $10 \%$ of NHS and $1 \%$ Penicillin/Streptomycin (SigmaAldrich) on 8-chamber culture slides (BD Biosciences Discovery Labware, Milan, Italy) to allow the adhesion of monocytes, which were left to differentiate for 7 days as reported above. Cells were incubated for 24 hours at $37^{\circ} \mathrm{C}$ and $5 \% \mathrm{CO}_{2}$ in RPMI-1640 GlutaMAX (Life Technologies) supplemented with $10 \%$ of GS and 1\% Penicillin/Streptomycin (SigmaAldrich) and subsequently stimulated for 3 hours with LPS $(100 \mathrm{ng} / \mathrm{mL})$ [8]. Macrophages were fixed and permeabilized with FIX \& PERM cell permeabilization kit (Società Italiana Chimici, Rome, Italy) according to the manufacturer's instructions and then blocked with human serum $(1: 60)$ for $30 \mathrm{~min}$ at RT. Afterwards, cells were incubated with primary mAb anti-CD68 (1:40) (Dako), mAb anti-CD206 (1:50) (Sigma-Aldrich), or rabbit anti-human PCT (Abcam) O/N at $4^{\circ} \mathrm{C}$. The binding of CD68 and CD206 antibodies was revealed with goat anti-mouse $\mathrm{Cy} 3$-conjugated secondary antibody $(1: 300)$, while biotinylated swine anti-rabbit $(1: 50)$ was followed by streptavidin-Cy2 $(1: 200)$ to detect the antiPCT polyAb, for 45 minutes in the dark at RT, followed by $4^{\prime}, 6$-diamidino-2-phenylindole (DAPI, Sigma-Aldrich) staining. Slides were prepared with mounting solution (Dako). Images were acquired with Leica DM3000 microscope (Leica, Wetzlar, Germany) and the pictures were collected using a Leica DFC320 digital camera (Leica).

2.8. Quantification of PCT Amount on the Culture Medium of Macrophages. Culture medium of macrophages was taken under sterile condition in a final volume of $150 \mu \mathrm{L} /$ sample, centrifuged at $12.000 \mathrm{~g}$ for 2 minutes at $+4^{\circ} \mathrm{C}$ to avoid the presence of cellular debris, and maintained at $-80^{\circ} \mathrm{C}$ until analysis. The amount of PCT in the culture medium of macrophages was quantified automatically by the Modular Analytics E170 Module (Roche Diagnostics, Milan, Italy) using BRAHMS PCT reagent (Roche, Mannheim, Germany).

\section{Statistical Analysis}

Statistical analysis was performed with Microsoft Office Excel 2003 (Microsoft Corporation, Redmond, CA, USA). Data were reported as mean \pm S.E.M. Mann-Whitney test was used to compare two groups of data and $P$ value of $<0.05$ was considered significant.

\section{Results}

4.1. Characterization of the Production of PCT in MO and M1 or M2 Polarized Macrophages. We initially investigated the ability of nonpolarized human macrophages (M0) to secrete PCT in the culture supernatant. The purity of human macrophages was confirmed with an immunofluorescence staining against the classical marker CD68 (Figure 1(a)). The levels of PCT were measured in the harvested supernatants of macrophages after 24 hours of culture. As shown in Figure 1(b), unstimulated macrophages produced about $20 \mathrm{pg} / \mathrm{mL}$ of PCT and no difference was observed in the supernatants of the cells stimulated with LPS.

The production of PCT by macrophages was also confirmed by the expression of the corresponding mRNA documented by qPCR (Figure 1(c)). The mRNA expression analysis revealed the presence of transcripts for PCT, confirming 


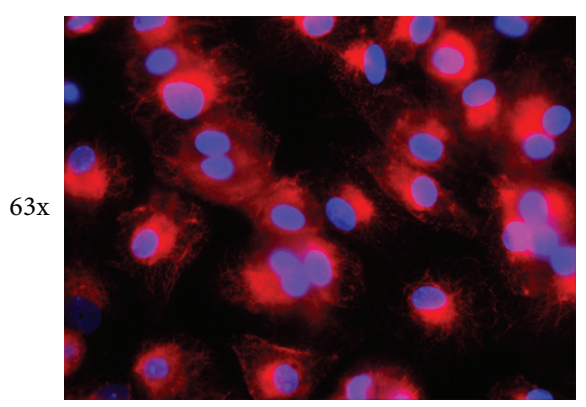

(a)

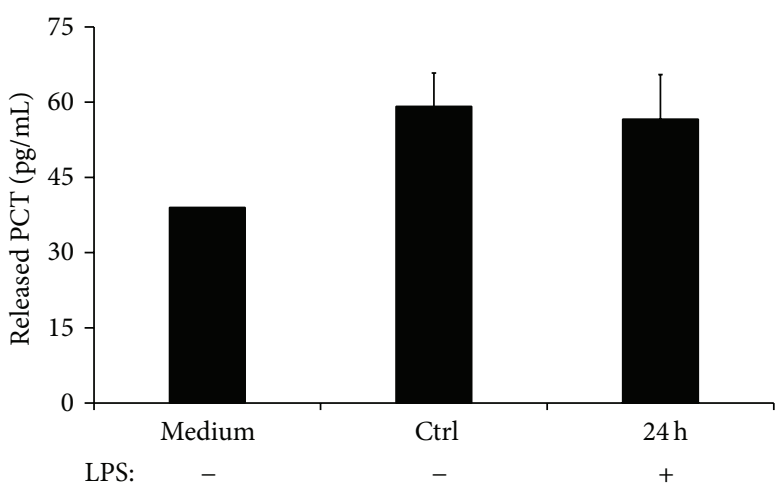

(b)

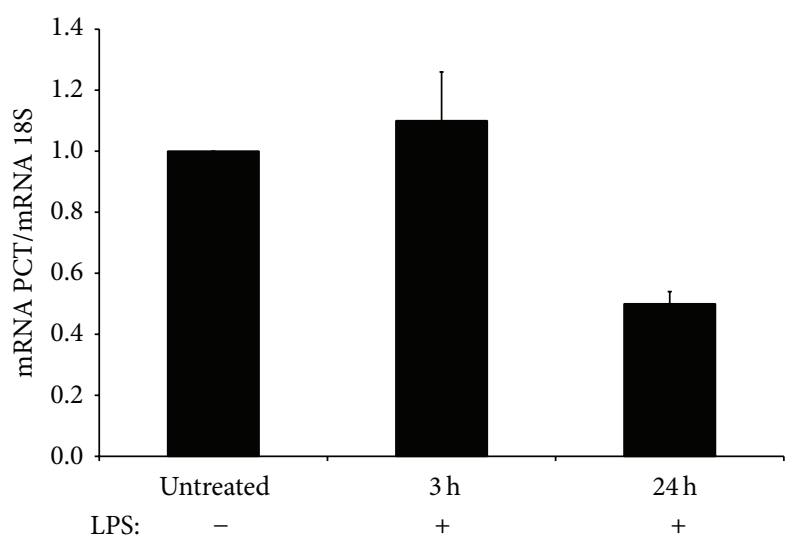

(c)
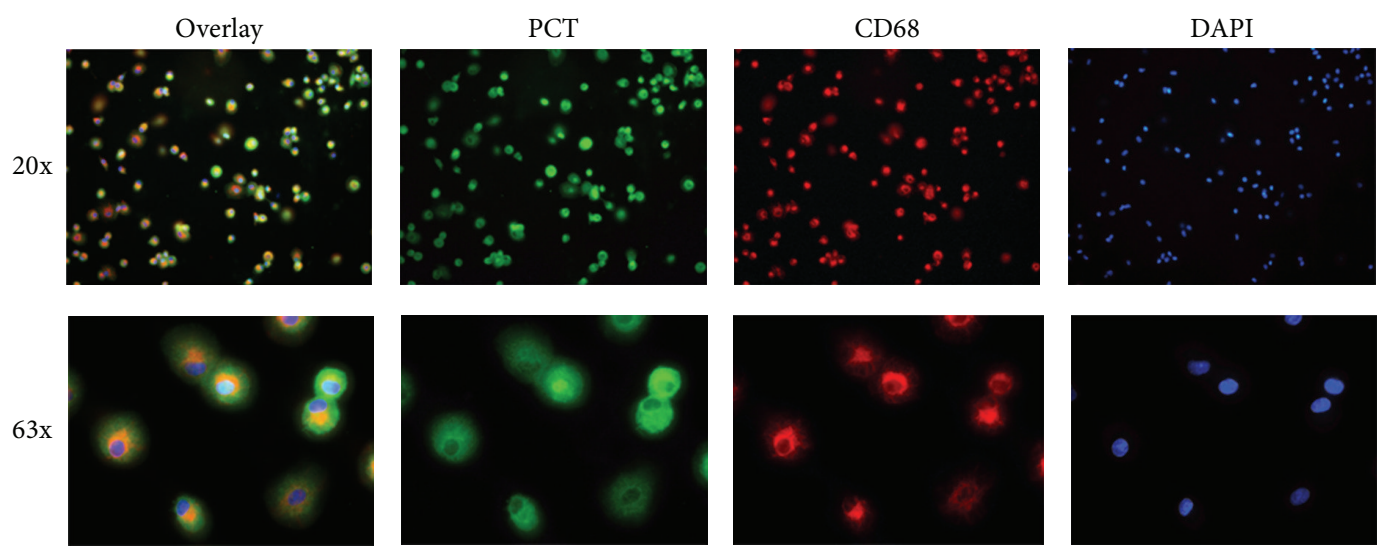

(d)

FIGURE 1: Human macrophages express PCT at mRNA and protein level. (a) Immunofluorescence analysis of human macrophages differentiated from PBMCs. Cells were stained with $\mathrm{mAb}$ anti-human CD68. DAPI nuclear staining is shown in blue. Original magnification 63x. (b) Levels of PCT secreted in the cell supernatant by cultured macrophages after 24 hours of stimulation with LPS. Data from 5 independent experiments are shown and no statistical significance has been found between treated and untreated cells. (c) RNA prepared from fully differentiated human macrophages, untreated or stimulated with LPS for 3 and 24 hours, were quantified for PCT and 18S expression by qPCR. Data from 5 independent experiments are shown and represent the mean \pm S.E.M. ${ }^{*} P<0.05$ (Mann-Whitney test). (d) Double Immunofluorescence analysis of human macrophages for PCT (green) and CD68 (red) expression. Overlay images show the PCT localized throughout the cytoplasm. DAPI nuclear staining is shown in blue. Original magnification 20x (upper panels) and 63x (lower panels). Images were acquired with Leica DM3000 microscope. 
the synthesis of this protein. In addition, cells stimulated with LPS for 3 hours did not reveal any modulation in mRNA expression. Unexpectedly, 24-hour stimulation with LPS induced a significant downregulation of the transcript for PCT. To further reveal the presence of PCT in human macrophages, a triple staining with DAPI and antibodies against PCT and CD68 was assessed on macrophages cultured in 8-chamber culture slides. PCT was clearly visible and localized throughout the cytoplasm as shown in Figure 1(d).

The cells were then polarized into fully differentiated M1 and M2 macrophages subtypes and characterized for the expression of some characteristic markers for the two subpopulations [26]. As shown in Figure 2(a), M1 macrophages showed upregulated gene expression of both the proinflammatory cytokine IL-1 $\beta$ and the cell surface marker CD80, while M2 macrophages, compared to M1 polarized or unstimulated macrophages (M0), increased the expression of the anti-inflammatory cytokine IL-10 and the mannose receptor C type 1 (CD206).

To assess if the polarization of the mononuclear phagocytes causes a different production of PCT, macrophages were then skewed towards their M1 and M2 phenotypes and stimulated for 3 hours with LPS to evaluate the modulation of the gene expression of PCT by qPCR. The synthesis of PCT was comparable between nonstimulated macrophages polarized towards their pro- and anti-inflammatory phenotypes compared to M0 macrophages. Indeed, the synthesis increased significantly in macrophages with M1 phenotype stimulated with LPS, compared to both M0 and M2 macrophages stimulated with the same concentration of the stimulus (Figure 2(b)). M1 macrophages were also shown to significantly upregulate (from 4.8 to $6 \mathrm{pg} / 10^{6}$ cells) the production of PCT following a stimulation with LPS for 24 hours, compared to those unstimulated with the same phenotype.

\subsection{Gravid Serum (GS) Induces an M2-Like Phenotype in} Cultured Macrophages. We hypothesized that the hormonal changes at the beginning of pregnancy may be able to modulate the phenotype of macrophages. To identify the effects of the first trimester gravid serum (GS) on the macrophage activation we evaluated the gene expression of some pro- and anti-inflammatory markers, modulated during the polarization of macrophages. As shown in Figure 3, we observed that 24 hours of culturing macrophages in the presence of GS significantly overexpressed the CD206 marker both at the gene and protein level. In the same experimental conditions, the expressions of IL-10 and IL- $1 \beta$ were, respectively, significantly upregulated and downregulated, meaning that the GS has the ability to skew macrophages through an anti-inflammatory phenotype. Interestingly, we showed that macrophages maintained the ability to respond to proinflammatory stimuli when conditioned with the GS, as they upregulated the expression of the IL- $1 \beta$ and TNF $\alpha$ cytokines in response to the stimulation with LPS for 3 hours (data not shown).
4.3. In Vitro Effects of the GS and the Pregnancy Hormones on PCT Expression in Cultured Macrophages. Based on the ability of the GS to polarize mononuclear phagocytes through an M2-like phenotype, we evaluated its effect on the expression of PCT. Macrophages cultured for 24 hours in the presence of GS and subsequently stimulated with LPS for 3 hours showed a significant reduction in PCT expression, compared to those cultured in NHS (Figure 4). Notably, the GS itself was able to downregulate the expression of PCT in macrophages during 24 hours of culture, compared to cells cultured in NHS, although the difference was not significant.

Furthermore, the hormonal milieu of the GS has been taken into account, trying to understand if it could be involved in the polarization of macrophages and if it is able to modulate both PCT and CD206 expression in these cells. Unexpectedly, the stimulation with progesterone (PG) induced a significant downregulation of PCT expression, while we observed an opposite effect of the $17 \beta$-Estradiol (E2). No modulation of the expression of PCT has been observed in macrophages stimulated with hCG. Interestingly, the three hormones together were not able to modulate PCT expression in macrophages, probably for the opposite effect of PG and E2 (Figure 5(b)). Note that all these hormones shared the ability to polarize macrophages through an M2like phenotype, as evidenced by the upregulation of the CD206 expression (Figure 5(a)).

\section{Discussion}

This is the first detailed study of PCT production by human macrophages, a powerful biomarker for an early and accurate diagnosis of bacterial infection [1].

The contribution of macrophages to PCT expression and secretion is not clear yet. The only study on the production of PCT by human macrophages in culture is by Linscheid et al. [8], which showed that macrophages at day 5 of culture did not express calcitonin or calcitonin gene-related peptide (CGRP)-I mRNA under basal conditions or after stimulation with several inflammatory mediators. The study demonstrated the ability of PBMCs to secrete PCT only after an adherence to endothelial cells or plastic surfaces. The induction was transient and it was not detectable after 18 hours of culture. Several studies investigated the production of PCT by PBMCs with contrasting results [1]. The presence of PCT has been previously observed by Oberhoffer and colleagues [11] in freshly isolated PBMCs both at transcriptional and translational level. Herget-Rosenthal et al. [27] have also demonstrated that PCT released by PBMCs, isolated from controls and patients with advanced chronic kidney disease, described a correlation between the PCT release from PBMCs and the concentration of PCT in the blood. Moreover, Balog et al. [10] showed that Gram-positive bacteria have the TNFinducing ability to elevate the intracellular content of PCT in human monocytes, although they hypothesized that other bacterial components can induce PCT directly. Müller et al. [5], in an animal model resembling human sepsis, found out that CT-mRNA was ubiquitously and uniformly expressed in 

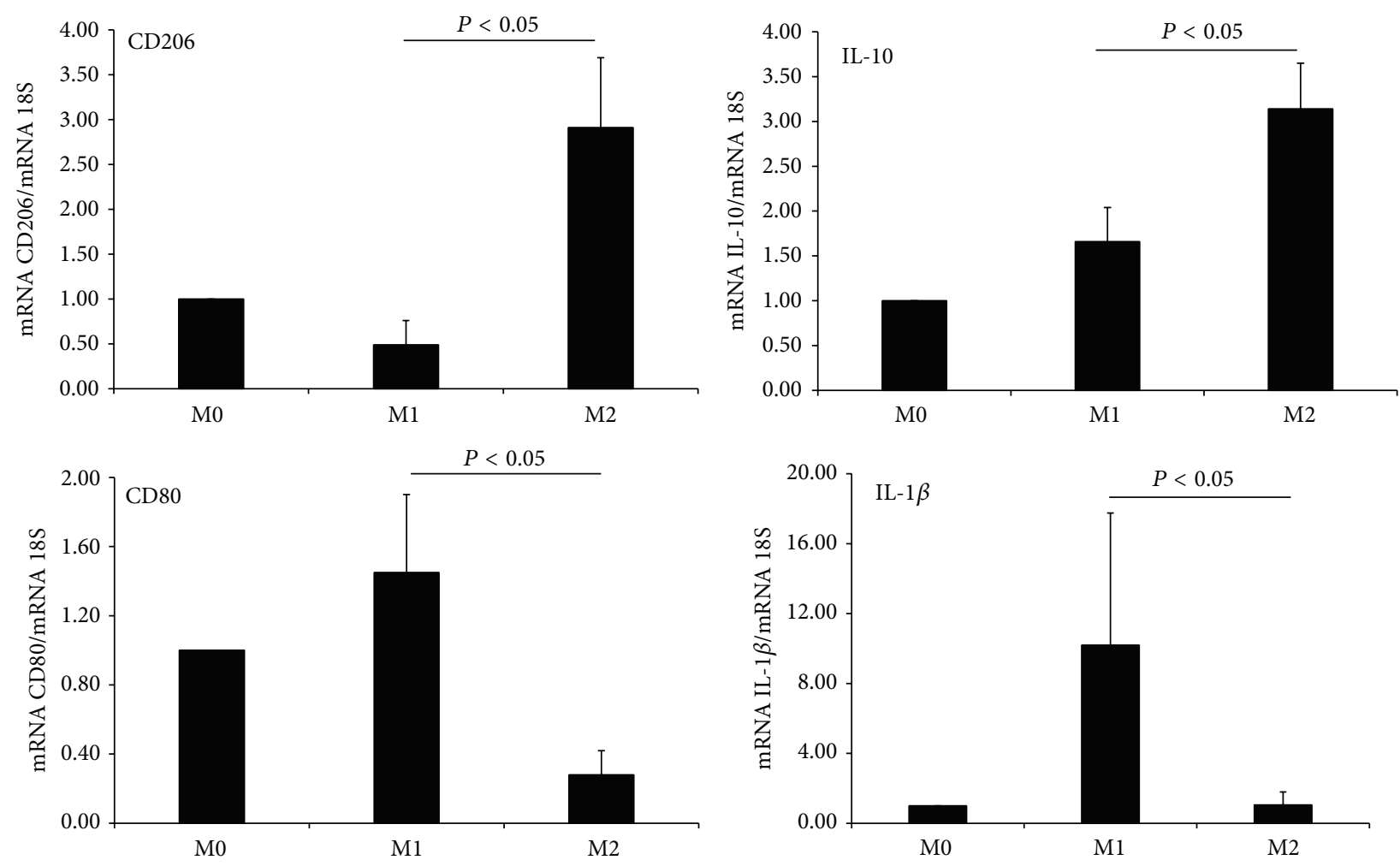

(a)

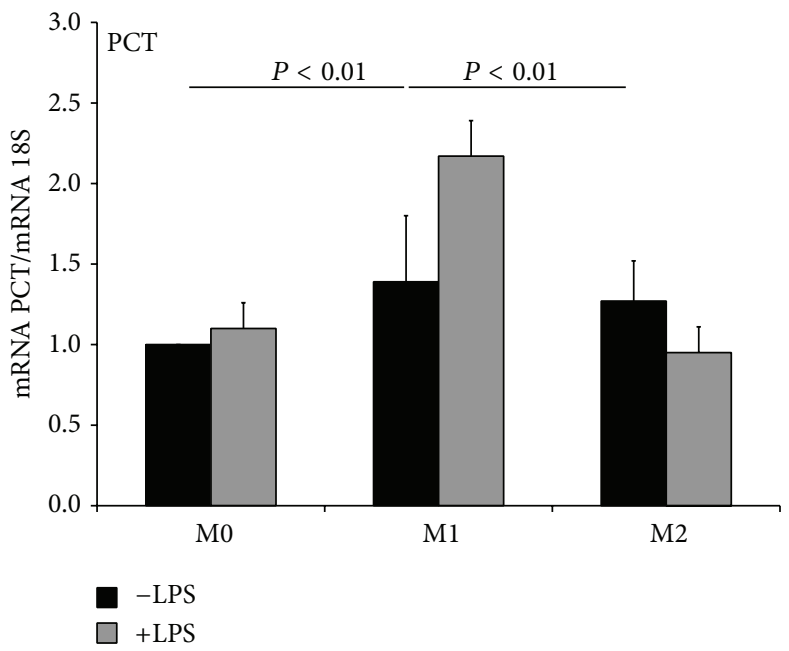

(b)

FIGURE 2: M1 polarized macrophages significantly upregulate the expression of PCT compared to both M0 and M2 macrophages under stimulation with LPS. (a) PMBCs were differentiated in culture for 7 days and polarized O/N with LPS (100 ng/mL) and IFN $\gamma(500 \mathrm{U} / \mathrm{mL})$ or IL-4 $(20 \mathrm{ng} / \mathrm{mL})$ and IL-10 $(50 \mathrm{ng} / \mathrm{mL})$ for the M1/M2 polarization. mRNA expression levels of CD80 and IL-1 $\beta$ (M1 markers) and CD206 and IL-10 (M2 markers) were measured by qPCR and normalized to those of the human housekeeping gene 18S. (b) RNA obtained from M0, M1, and M2 macrophages were quantified for PCT and 18S expression by qPCR. The relative amount of mRNA for PCT was normalized with reference to $18 \mathrm{~S}$ value. Results were expressed as AUs, in which $1 \mathrm{AU}$ represents the value obtained with untreated macrophages used as a positive control. Bars represent the mean \pm S.E.M. of at least 3 independent experiments. ${ }^{*} P<0.05$ and ${ }^{* *} P<0.01$ (Mann-Whitney test).

multiple tissues throughout the body in response to sepsis, including peritoneal macrophages.

Our study demonstrates the ability of cultured human macrophages to produce PCT after 7 days of culture. Furthermore, we investigated the effect of LPS in macrophage production of PCT. Our data revealed that human macrophages constitutively produce PCT under basal conditions, but are unable to respond to LPS in terms of PCT expression and production. The main and novel finding of the present study is that the ability to 

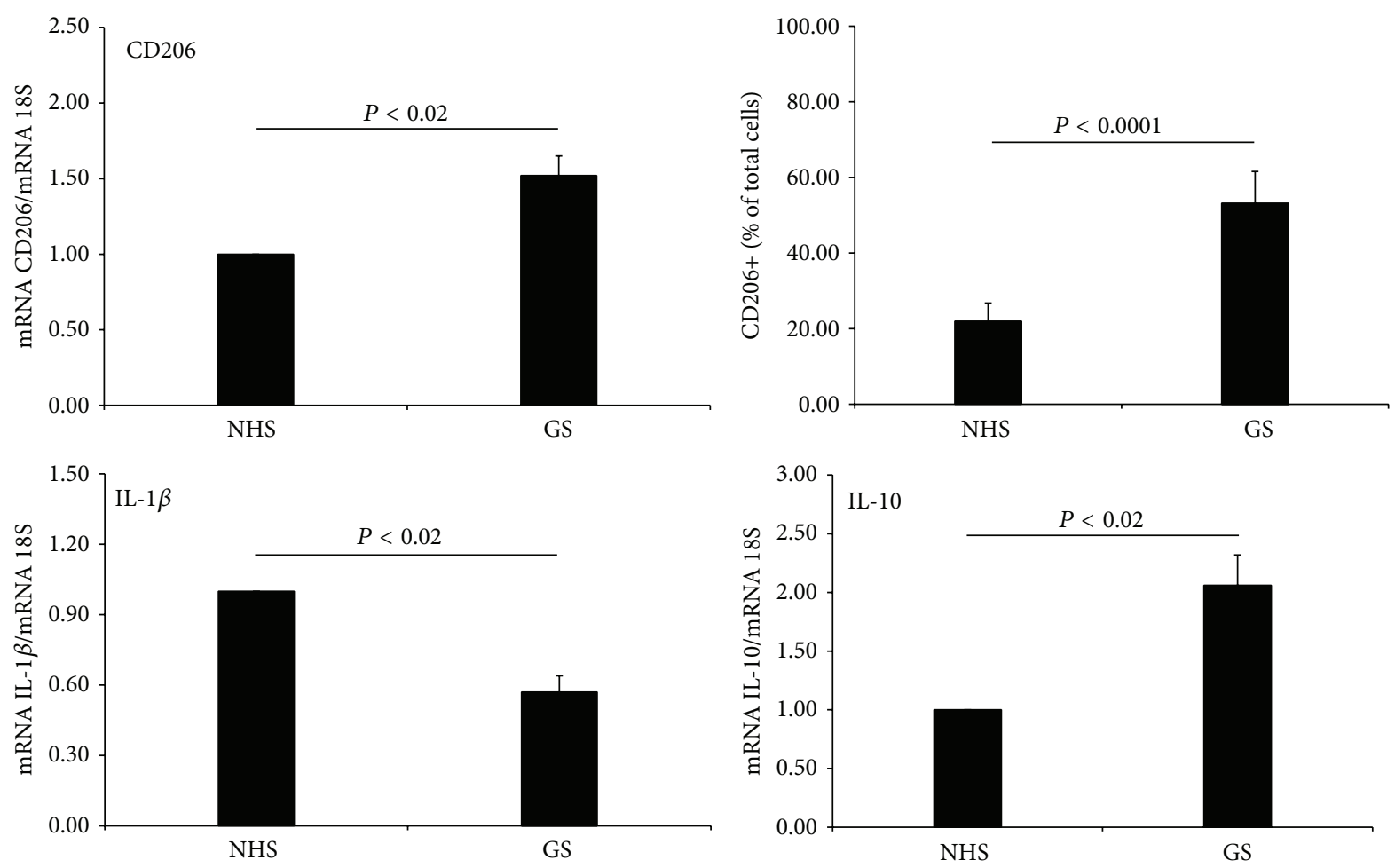

(a)
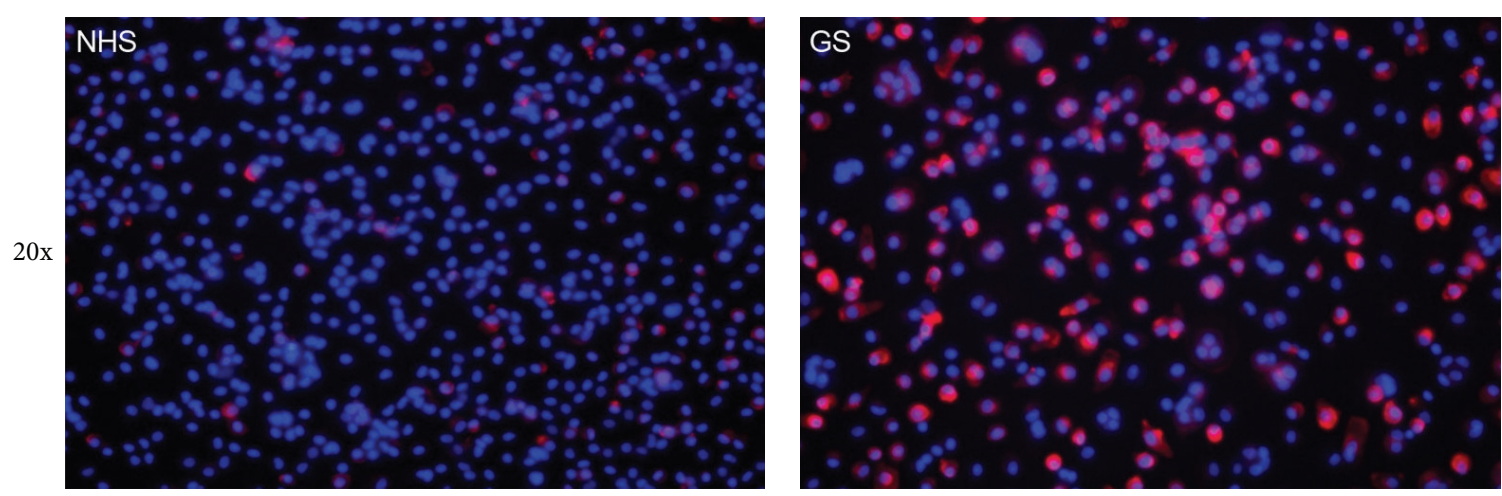

(b)

FIGURE 3: Human macrophages conditioned for 24 hours with gravid serum (GS) downregulate the expression of IL-1 $\beta$ and upregulate IL-10. Increased expression of CD206 was observed at gene and protein level. (a) mRNA expression levels of IL-1 $\beta$, IL-10, and CD206 were measured by qPCR and normalized to those of the human housekeeping gene 18S. Results were expressed as AUs, in which 1 AU represents the value obtained with macrophages cultured in NHS used as a positive control. The amount of CD206 positive macrophages was evaluated by counting labeled cells using ImageJ (NIH, United States), considering a percentage of positive cells in at least 5 different fields. Bars represent the mean \pm S.E.M. of at least 3 independent experiments. ${ }^{*} P<0.02$ and ${ }^{* * *} P<0.0001$ (Mann-Whitney test). (b) Immunofluorescence reveals the upregulation of the expression of CD206 protein (red) in human macrophages cultured for 24 hours in presence of GS. DAPI nuclear staining is shown in blue. Images were acquired with Leica DM3000 microscope. Original magnification 20x.

increase the production of PCT after LPS stimulation is acquired when macrophages have been skewed towards their M1 phenotype. On the contrary, no modulation in PCT expression was observed in M2 macrophages stimulated with the same amount of LPS. These data are interesting, since M1 macrophages are generally considered responsible for resistance against intracellular pathogens and are associated with acute bacterial infections and sepsis [18]. Furthermore, based on its structure, it has recently been shown that PCT can interact with bacterial LPS inducing a decrease of its proinflammatory effects [21]. These data indicate that the peculiar ability of M1 to produce PCT is probably associated with their physiological function of defence.

The other important observation of our study is that macrophages cultured in GS, obtained from women during the first trimester of pregnancy, skewed towards an M2like phenotype and consequently, when stimulated with LPS, significantly decreased the level of mRNA for PCT. In an 


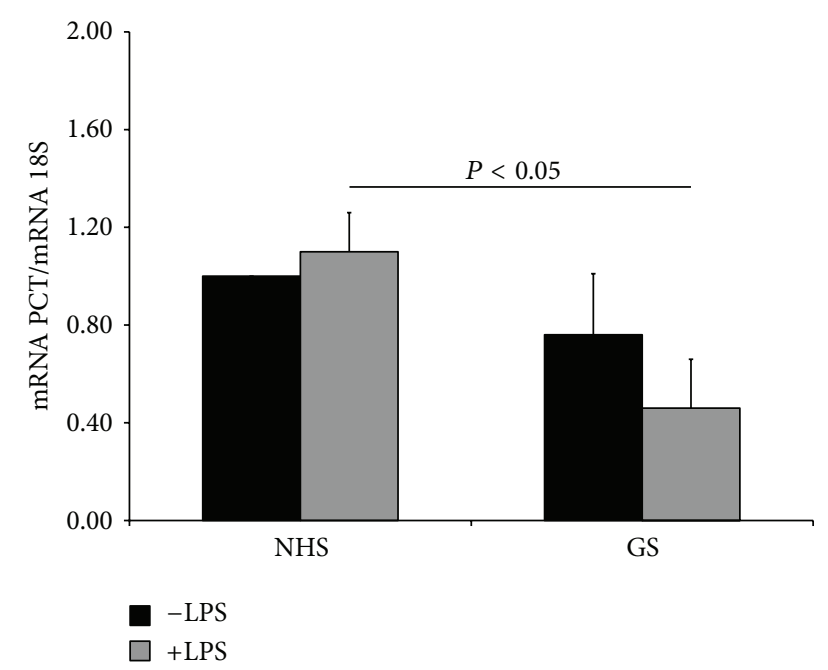

FIGURE 4: The expression of PCT is negatively regulated in human macrophages conditioned for 24 hours with gravid serum (GS), reaching significance after stimulation with $100 \mathrm{ng} / \mathrm{mL}$ of LPS for 3 hours. mRNA expression levels of these proteins were measured by qPCR and normalized to those of the human housekeeping gene 18S. Results were expressed as AUs, in which $1 \mathrm{AU}$ represents the value obtained with macrophages cultured in NHS used as a positive control. Bars represent the mean \pm S.E.M. of at least 3 independent experiments. ${ }^{*} P<0.05$ (Mann-Whitney test).

attempt to identify the factors present in GS serum, able to modulate the expression of PCT in human macrophages, we evaluated the effect of PG, E2, and hCG. Our data showed that hCG has no effect on, while PG downregulates and E2 increases the PCT expression after LPS stimulation. The fact that the combined stimulation with three hormones does not have an impact on the modulation of the expression of PCT indicates that additional factors present in the GS, for example, cytokines such as IL-10 [28], play a role in the regulation of the production of this protein in macrophages. We can speculate that all tissue macrophages change their phenotype getting in contact with serum derived factors during pregnancy. Taking into account that placental-derived components in the GS are more concentrated in the placental milieu, we can hypothesize that the phenotype of macrophages obtained in our experiments resembles more those present at the fetal-maternal interface.

Very recent data from Koldehoff et al. [29] demonstrated that the gene profile of monocytes during the first trimester pregnancy differs from the one of monocytes isolated from nonpregnant women. On the basis of these data, we can assume that circulating monocytes may be primed by GS factors too, although this issue needs to be further analyzed.

Pregnancy is associated with a unique immunological condition, characterized by decidual, as well as peripheral immune responses adaptation, in order to guarantee maternal tolerance to the foetus [30]. It has been demonstrated that decidual macrophages express markers of alternative activation, including CD206 and IL-10 [23]. Furthermore, it has been previously shown that soluble circulating factors, present in sera of pregnant women, induce a dendritic

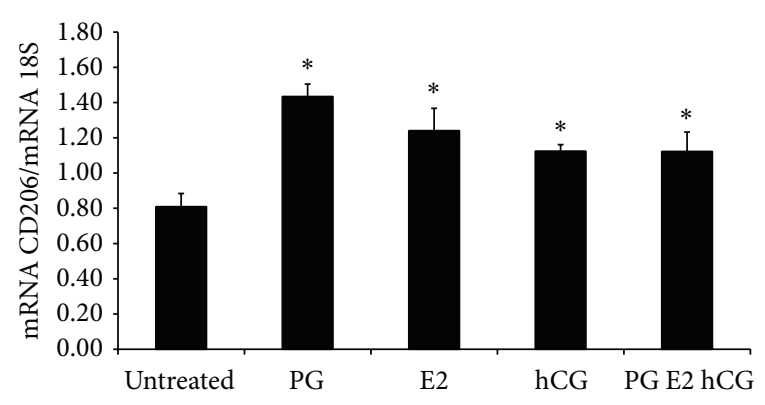

(a)

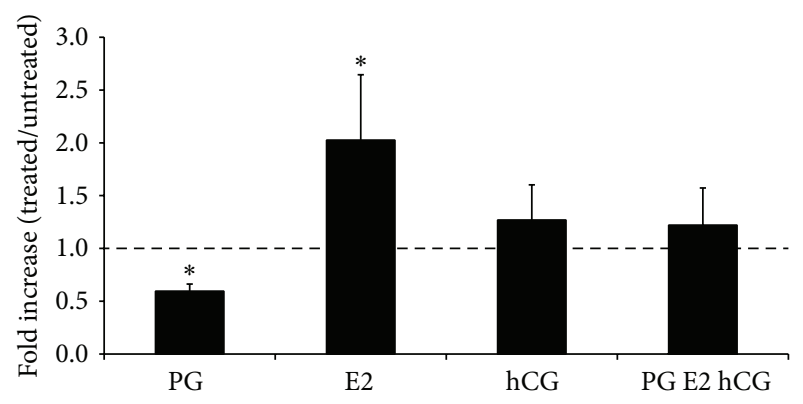

(b)

FIGURE 5: Expression of CD206 and PCT in human macrophages conditioned for 24 hours with PG, E2, and hCG or with the three hormones combined together. (a) The mRNA expression level of CD206 was measured by qPCR and normalized to those of the human housekeeping gene 18S. Results were expressed as AUs, in which $1 \mathrm{AU}$ represents the value obtained with macrophages cultured in NHS used as a positive control. (b) PCT fold increase was expressed as ratio between mRNA levels of LPS stimulated and LPS untreated cells. Bars represent the mean \pm S.E.M. of at least 3 independent experiments. ${ }^{*} P<0.05$ (Mann-Whitney test).

cell (DC) incomplete activation that was associated with reduced DC allostimulatory capacity [31]. In this study we demonstrated that during human physiological pregnancy macrophages, in response to human pregnant sera, also undergo profound changes that probably reflect the maternal systemic reaction to the foetus. Our data showed that also the synthesis of PCT is strongly downregulated in macrophages with this particular phenotype, although the response to LPS is conserved in terms of production of the proinflammatory cytokine IL-1 $\beta$ (data not shown). These data are in agreement with Mor statements [30], which indicate that the immune system at the implantation site is not suppressed but functionally and carefully controlled. The fact that the expression of PCT is downregulated indicates that it may probably have harmful effects to fetal development and pregnancy progression.

\section{Conclusion}

In conclusion, our results demonstrated that the expression and synthesis of PCT after LPS stimulation are a peculiar ability of M1 macrophages. Furthermore, we found out that macrophages cultured in first trimester GS acquire an M2like phenotype, not allowing them to produce PCT after 
LPS stimulation. Based on these data, we can speculate that pregnant women might respond less efficiently to sepsis than nonpregnant women in terms of about $50 \%$ reduction of PCT expression in macrophages. Our data, although they were obtained only in an in vitro model of cultured human macrophages, indicate that the slowdown of PCT synthesis might have an impact on the use of this laboratory marker for the analysis of the inflammatory status in pregnant women during sepsis and in autoimmune and inflammatory diseases.

Above all, these data support the concept reported by Mor and Cardenas [30] that pregnancy is a unique condition, in which the immune system is modulated, but not suppressed, leading to a differential response against bacterial infections, which is not completely known yet.

\section{Conflict of Interests}

The authors declare that there is no conflict of interests regarding the publication of this paper.

\section{Authors' Contribution}

Oriano Radillo and Roberta Bulla share senior authorship.

\section{Acknowledgments}

The authors thank Dr. Luca Mascaretti, Centro Trasfusionale Ospedale Maggiore di Trieste (Trieste, Italy), for providing buffy coats from healthy blood donors and Giulia Guzzetti for her helpful revision of the paper. This work was supported by a research grant of the Ministry of Health (Ricerca Finalizzata RC 34/11), grants from European Network of Excellence "EMBIC" within FP6 (Contract no. LSHNCT-2004512040), Italian Association for Cancer Research (AIRC), Italian Ministry of University and Research (PRIN MFXE7L), Region Friuli-Venezia Giulia (LINFONET and AITT art. 23 legge regionale 26/2005), and Fondazione Casali-Trieste (to Roberta Bulla).

\section{References}

[1] G. N. Matwiyoff, J. D. Prahl, R. J. Miller et al., "Immune regulation of procalcitonin: a biomarker and mediator of infection," Inflammation Research, vol. 61, no. 5, pp. 401-409, 2012.

[2] M. G. Rosenfeld, J. J. Mermod, S. G. Amara et al., "Production of a novel neuropeptide encoded by the calcitonin gene via tissuespecific RNA processing," Nature, vol. 304, no. 5922, pp. 129$135,1983$.

[3] K. L. Becker, R. Snider, and E. S. Nylen, "Procalcitonin in sepsis and systemic inflammation: a harmful biomarker and a therapeutic target," British Journal of Pharmacology, vol. 159, no. 2, pp. 253-264, 2010.

[4] D. N. Gilbert, "Use of plasma procalcitonin levels as an adjunct to clinical microbiology," Journal of Clinical Microbiology, vol. 48, no. 7, pp. 2325-2329, 2010.

[5] B. Müller, J. C. White, E. S. Nylén, R. H. Snider, K. L. Becker, and J. F. Habener, "Ubiquitous expression of the calcitonin-I gene in multiple tissues in response to sepsis," The Journal of Clinical Endocrinology \& Metabolism, vol. 86, no. 1, pp. 396-404, 2001.
[6] S. Russwurm, I. Stonans, E. Stonane et al., "Procalcitonin and CGRP-I mRNA expression in various human tissues," Shock, vol. 16, no. 2, pp. 109-112, 2001.

[7] P. Linscheid, D. Seboek, E. S. Nylen et al., "In vitro and in vivo calcitonin I gene expression in parenchymal cells: a novel product of human adipose tissue," Endocrinology, vol. 144, no. 12, pp. 5578-5584, 2003.

[8] P. Linscheid, D. Seboek, D. J. Schaer, H. Zulewski, U. Keller, and B. Müller, "Expression and secretion of procalcitonin and calcitonin gene-related peptide by adherent monocytes and by macrophage-activated adipocytes," Critical Care Medicine, vol. 32, no. 8, pp. 1715-1721, 2004.

[9] P. Dandona, D. Nix, M. F. Wilson et al., "Procalcitonin increase after endotoxin injection in normal subjects," The Journal of Clinical Endocrinology \& Metabolism, vol. 79, no. 6, pp. 16051608, 1994.

[10] A. Balog, I. Ocsovszki, and Y. Mándi, "Flow cytometric analysis of procalcitonin expression in human monocytes and granulocytes," Immunology Letters, vol. 84, no. 3, pp. 199-203, 2002.

[11] M. Oberhoffer, I. Stonans, S. Russwurm et al., "Procalcitonin expression in human peripheral blood mononuclear cells and its modulation by lipopolysaccharides and sepsis-related cytokines in vitro," The Journal of Laboratory and Clinical Medicine, vol. 134, no. 1, pp. 49-55, 1999.

[12] D. N. Gilbert, "Procalcitonin as a biomarker in respiratory tract infection," Clinical Infectious Diseases, vol. 52, supplement 4, pp. S346-S350, 2011.

[13] M. Meisner, Ed., Procalcitonin-Biochemical and Clinical Diagnosis, International Medical Publishers, London, UK, 2012.

[14] J. W. Pollard, "Trophic macrophages in development and disease," Nature Reviews Immunology, vol. 9, no. 4, pp. 259-270, 2009.

[15] F. Geissmann, S. Gordon, D. A. Hume, A. M. Mowat, and G. J. Randolph, "Unravelling mononuclear phagocyte heterogeneity, Nature Reviews Immunology, vol. 10, no. 6, pp. 453-460, 2010.

[16] A. Mantovani, A. Sica, and M. Locati, "Macrophage polarization comes of age," Immunity, vol. 23, no. 4, pp. 344-346, 2005.

[17] A. Mantovani, A. Sica, and M. Locati, "New vistas on macrophage differentiation and activation," European Journal of Immunology, vol. 37, no. 1, pp. 14-16, 2007.

[18] A. Sica and A. Mantovani, "Macrophage plasticity and polarization: in vivo veritas," The Journal of Clinical Investigation, vol. 122, no. 3, pp. 787-795, 2012.

[19] T. Nagamatsu and D. J. Schust, "The immunomodulatory roles of macrophages at the maternal-fetal interface," Reproductive Sciences, vol. 17, no. 3, pp. 209-218, 2010.

[20] R. Bulla, F. Bossi, and F. Tedesco, "The complement system at the embryo implantation site: friend or foe?" Frontiers in Immunology, vol. 3, no. 55, pp. 1-8, 2012.

[21] G. Matera, A. Quirino, A. Giancotti et al., "Procalcitonin neutralizes bacterial LPS and reduces LPS-induced cytokine release in human peripheral blood mononuclear cells," $B M C$ Microbiology, vol. 12, article 68, 2012.

[22] G. Solinas, S. Schiarea, M. Liguori et al., "Tumor-conditioned macrophages secrete migration-stimulating factor: a new marker for M2-polarization, influencing tumor cell motility," The Journal of Immunology, vol. 185, no. 1, pp. 642-652, 2010.

[23] J. Svensson, M. C. Jenmalm, A. Matussek, R. Geffers, G. Berg, and J. Ernerudh, "Macrophages at the fetal-maternal interface 
express markers of alternative activation and are induced by MCSF and IL-10," The Journal of Immunology, vol. 187, no. 7, pp. 3671-3682, 2011.

[24] R. Bulla, C. Agostinis, F. Bossi et al., "Decidual endothelial cells express surface-bound Clq as a molecular bridge between endovascular trophoblast and decidual endothelium," Molecular Immunology, vol. 45, no. 9, pp. 2629-2640, 2008.

[25] R. D. McCurdy, J. J. McGrath, and A. Mackay-Sim, "Validation of the comparative quantification method of real-time PCR analysis and a cautionary tale of housekeeping gene selection," Gene Therapy and Molecular Biology, vol. 12, no. 1, pp. 15-24, 2008.

[26] C. A. Ambarus, S. Krausz, M. van Eijk et al., "Systematic validation of specific phenotypic markers for in vitro polarized human macrophages," Journal of Immunological Methods, vol. 375, no. 1-2, pp. 196-206, 2012.

[27] S. Herget-Rosenthal, T. Klein, G. Marggraf et al., "Modulation and source of procalcitonin in reduced renal function and renal replacement therapy," Scandinavian Journal of Immunology, vol. 61, no. 2, pp. 180-186, 2005.

[28] A. Kumar, N. Begum, S. Prasad, S. Agarwal, and S. Sharma, "IL10 , TNF- $\alpha$ \& IFN- $\gamma$ : potential early biomarkers for preeclampsia," Cellular Immunology, vol. 283, no. 1-2, pp. 70-74, 2013.

[29] M. Koldehoff, B. Cierna, N. K. Steckel, D. W. Beelen, and A. H. Elmaagacli, "Maternal molecular features and gene profiling of monocytes during first trimester pregnancy," Journal of Reproductive Immunology, vol. 99, no. 1, pp. 62-68, 2013.

[30] G. Mor and I. Cardenas, "The immune system in pregnancy: a unique complexity," American Journal of Reproductive Immunology, vol. 63, no. 6, pp. 425-433, 2010.

[31] S. D. Bella, S. Giannelli, V. Cozzi et al., "Incomplete activation of peripheral blood dendritic cells during healthy human pregnancy," Clinical \& Experimental Immunology, vol. 164, no. 2, pp. 180-192, 2011. 


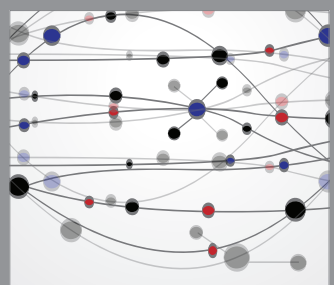

The Scientific World Journal
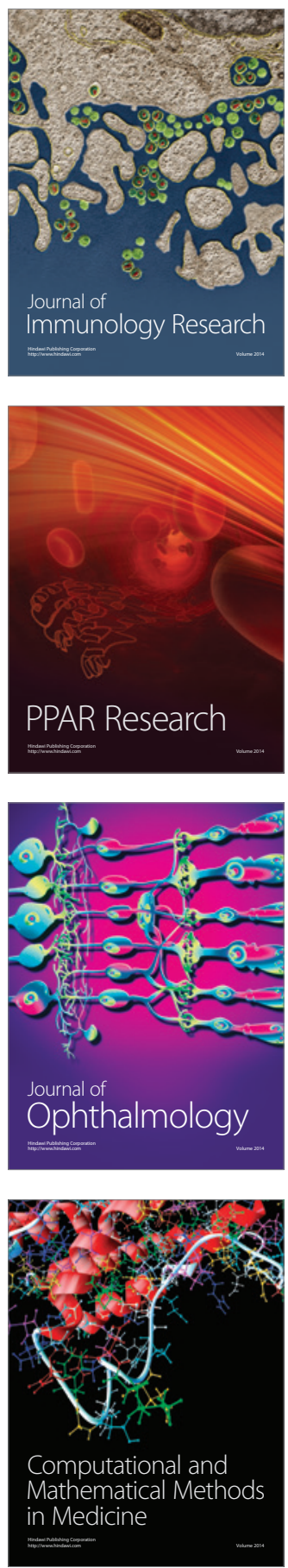

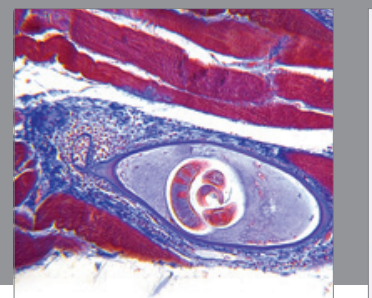

Gastroenterology

Research and Practice
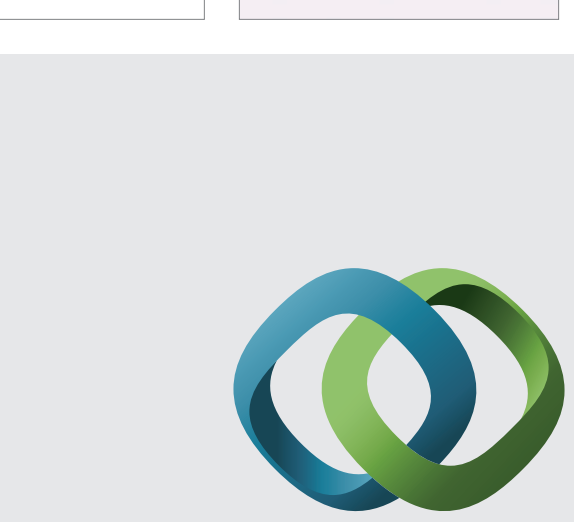

\section{Hindawi}

Submit your manuscripts at

http://www.hindawi.com
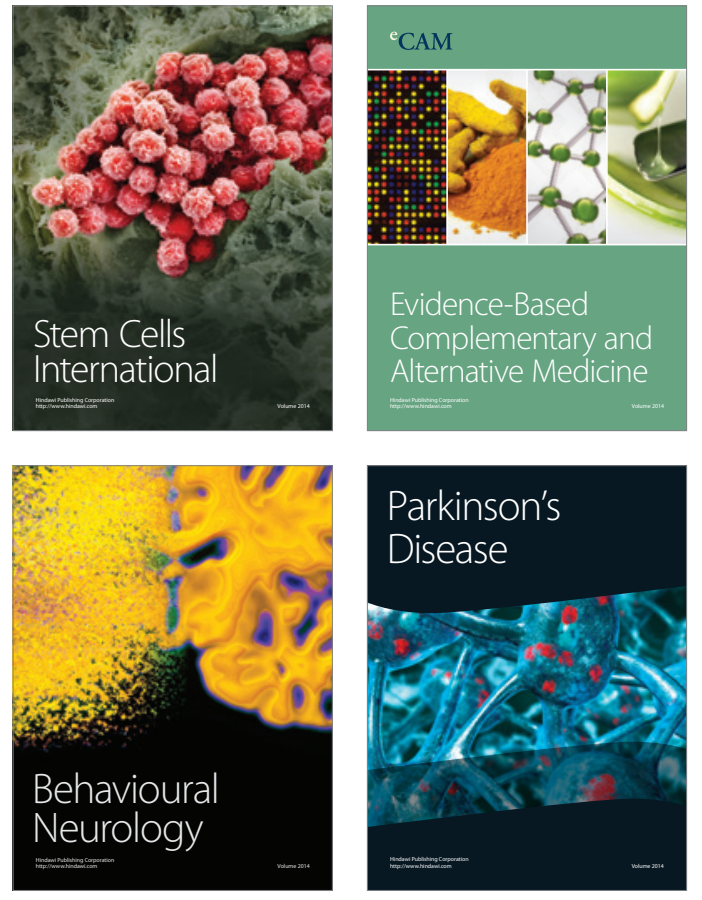
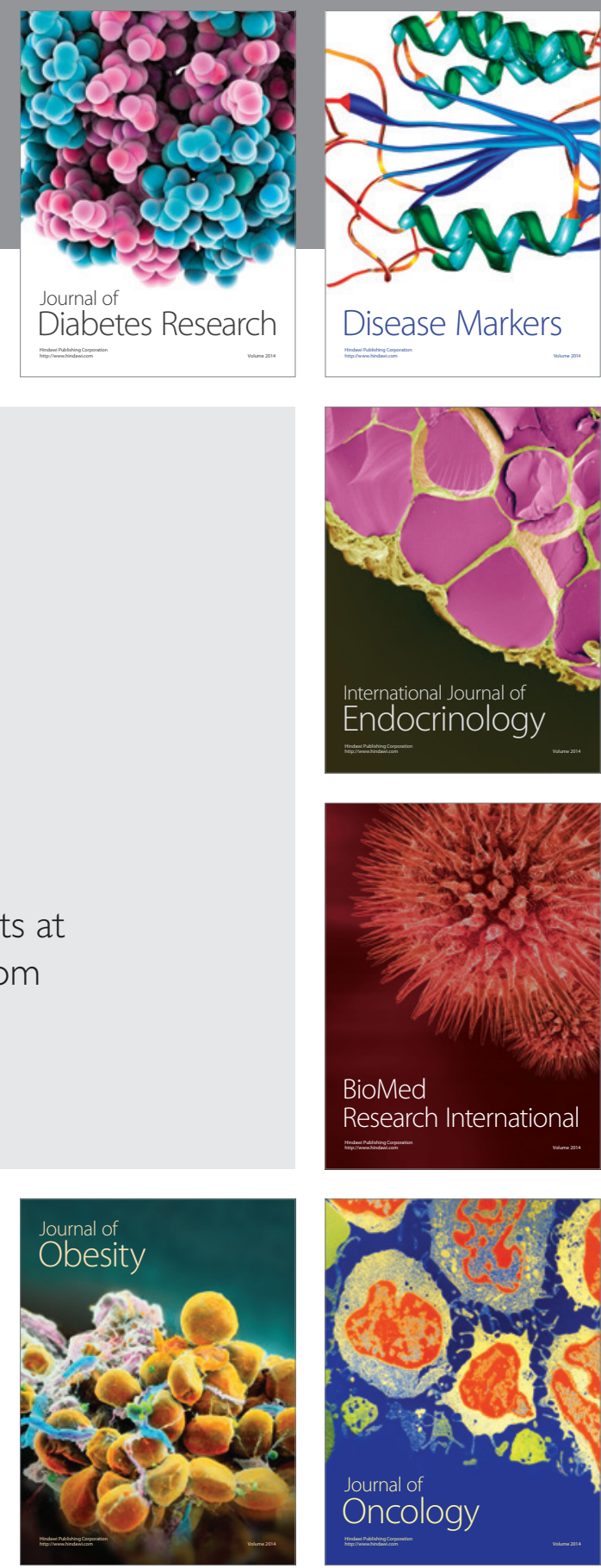

Disease Markers
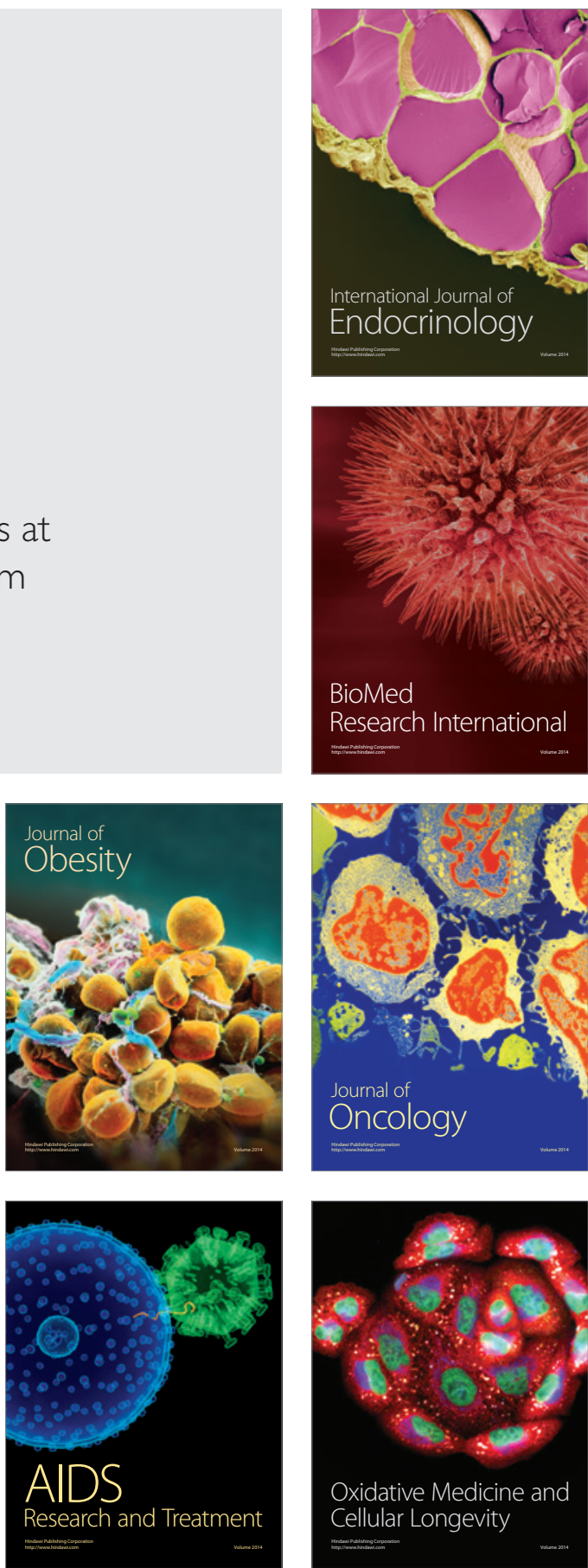